\title{
RESET
}

Recherches en sciences sociales sur Internet

$10 \mid 2021$

Savoirs incertains

\section{Dévoiler les « bobards » des médias dominants}

Les stratégies de (dé)légitimation de la réinformation

Exposing the "lies" of mainstream media. The "reinformation" movement's

(de)legitimization strategies

\section{Gaël Stephan et Ysé Vauchez}

\section{(2) OpenEdition \\ Journals}

Édition électronique

URL : https://journals.openedition.org/reset/3180

DOI : $10.4000 /$ reset.3180

ISSN : 2264-6221

\section{Éditeur}

Association Recherches en sciences sociales sur Internet

\section{Référence électronique}

Gaël Stephan et Ysé Vauchez, "Dévoiler les «bobards » des médias dominants », RESET [En ligne],

10 | 2021, mis en ligne le 13 mai 2021, consulté le 22 mai 2021. URL : http://journals.openedition.org/ reset/3180 ; DOI : https://doi.org/10.4000/reset.3180

Ce document a été généré automatiquement le 22 mai 2021.

(c) Association Recherches en sciences sociales sur Internet 


\title{
Dévoiler les « bobards » des médias dominants
}

\author{
Les stratégies de (dé)légitimation de la réinformation \\ Exposing the "lies" of mainstream media. The "reinformation" movement's \\ (de)legitimization strategies
}

Gaël Stephan et Ysé Vauchez

\section{Introduction}

1 Historiquement, l'extrême droite s'est appuyée sur de multiples titres de presse, incarnant les différentes tendances qui la composent (Camus \& Monzat, 1992). Aussi une galaxie de journaux, bulletins, lettres et autres revues constitue-t-elle l'un des principaux piliers de la culture d'extrême droite en France (Camus, 1996). Si des contrediscours relatifs à l'actualité existaient donc avant l'émergence d'Internet, le réseau des réseaux représente cependant une opportunité discursive nouvelle. Pour reprendre à notre compte l'expression de F. Rebillard (2007), internet a représenté une accélération - abaissant les coûts de production et de diffusion, allégeant les contraintes éditoriales, etc. - plus qu'une mise en mouvement pour la presse d'extrême droite.

Le développement d'un médiactivisme ${ }^{1}$ (Cardon \& Granjon, 2010) extrême droitier en ligne est un phénomène qui traverse plusieurs pays occidentaux (Figenschou \& Ihlebaek, 2018), et est conduit en France et dans les pays francophones sous le nom de "réinformation». Le terme est notamment promu par J.-Y. Le Gallou ${ }^{2}$ et sa fondation identitaire Polémia : il s'agit, selon leur propre expression, de s'opposer aux médias «du système $»^{3}$ que la soumission aux diktats du politiquement correct rendrait coupables de désinformation, en créant des médias alternatifs, censés rétablir la vérité en se soustrayant à la pensée unique. Ce sont les stratégies développées par ces "médiactivistes de l'information" que ce travail propose d'analyser, à travers une analyse ethnographique et thématique des "Bobards d'Or», cérémonie annuelle 
organisée à Paris par Polémia, qui constitue un moment de sociabilité hors-ligne important pour le mouvement.

3 Si les frontières du courant de la réinformation sont difficilement délimitables l'ensemble étant constitué d'un chapelet de sites aux niveaux de développement et d'activité éditoriale variables - quelques études monographiques ont été menées sur des sites ayant en commun de développer une critique des médias mainstream. À titre d'exemple, le site Les Observateurs propose à ses internautes une "vision de l'actualité suisse libérée de la pensée unique », selon les termes de ses animateurs, et présente une ligne éditoriale articulée autour de la valorisation du souverainisme, de la crainte d'une « islamisation de l'Europe », et de la dénonciation des «mensonges des médias » (Jammet \& Guidi, 2017 : 245). Le blog Fdesouche, est lui un infomédiaire (au sens de Rebillard \& Smyrnaios, 2010) qui organise son offre autour de trois thématiques: immigration, islam et insécurité. Il s'attaque à la crédibilité des "médias dominants » ${ }^{4}$ en reprenant des contenus produits par ces derniers et en les mettant en concurrence avec des sources illégitimes eu égard aux standards journalistiques, notamment des publications issues des réseaux socio-numériques (Lukasik, 2018). Ce faisant, les animateurs du blog affirment la fin du monopole des journalistes mainstream sur la mise en mots et en images de l'actualité. Ces exemples ne doivent pas conduire à penser que la réinformation ne se déploie qu'en ligne : y sont également associés le quotidien Présent et Radio Courtoisie, formes non-numériques d'hybridation entre journalisme et militantisme.

\section{Terrain et méthodologie}

4 L'enquête présentée ici est fondée sur le croisement de différentes méthodes appliquées à un même objet, la cérémonie des Bobards d'Or, à travers des approches à la fois quantitatives et qualitatives, en ligne et hors ligne. Récompensant "[les journalistes] qui n'hésitent pas à mentir délibérément pour servir le politiquement correct $~^{5}$, cet événement contribue à la construction et au renforcement de l'image des médias comme affabulateurs. C'est sa fréquentation qui nous a permis d'entrer en contact avec les médiactivistes de réinformation.

Créée par la fondation Polémia en 2010, la cérémonie se déroule depuis 2016 au Théâtre du Gymnase Marie Bell (800 places), à Paris. Elle fait l'objet d'une captation vidéo, étant diffusée sur des sites de réinformation partenaires de l'évènement. Les vidéos des éditions passées sont toujours consultables en ligne car archivées sur différentes plateformes. La sélection des mensonges primés à l'occasion de la cérémonie s'opère en deux temps. Des prises de positions de journalistes estimées ridicules ou scandaleuses, et des événements dont la couverture est jugée erronée ou biaisée (les « bobards ») sont mis en ligne sur le site internet de la cérémonie. Les internautes sont appelés à choisir ceux qu'ils estiment les plus ridicules, qui sont alors soumis au vote à main levée des spectateurs de la cérémonie. À cette occasion, chaque «bobard» est présenté et défendu par un juré, qui représente la rédaction d'un média de réinformation. La cérémonie est aussi émaillée de saynètes, de présentations de vidéos ou de caricatures qui renforcent sa prétention humoristique.

6 Nous nous sommes chacun rendus à la cérémonie à des éditions différentes, celle-ci étant ouverte à tous, à condition de s'acquitter d'une entrée (qui reste gratuite pour les moins de 25 ans). Si nos voisins, dans la queue, dans l'attente de l'ouverture des portes 
du théâtre, ou dans la corbeille, n'ont manifesté aucune curiosité à notre égard, plusieurs éléments nous renvoyaient à notre statut de «touristes » (Unruh, 1980) dans ce monde sur lequel nous travaillons, sans en maitriser l'intégralité des codes. Le premier indice de notre statut d'observateur est notre absence de connaissances dans le public, ce qui apparaît de manière flagrante dans la queue, et de manière moindre à la fin de la cérémonie. Alors que l'attente est l'occasion pour certains des membres du public, et en particulier ceux de notre classe d'âge, de retrouver des connaissances, l'observateur extérieur est évidemment tenu écarté de ce jeu de salutations, remarques amicales et sourires de connivence. Le second élément renseignant notre statut est notre tenue vestimentaire. Malgré des efforts, en préparation de la soirée, nous avons chacun de notre côté la sensation de ne pas avoir appréhendé correctement les codes vestimentaires en vigueur. Ceci est d'autant plus prononcé pour notre observatrice : en portant un pantalon, elle sent qu'elle tranche avec les quelques jeunes femmes, et surtout les femmes plus âgées à l'éthos bourgeois. Habillées de robes, maquillées, portant pour certaines des bijoux d'apparat ou des toques en vison, ces femmes nous renvoient à une dimension que seule l'observation in situ révèle: les Bobards d'Or ne sont pas uniquement un prétexte pour réunir des médiactivistes de réinformation, ils sont un spectacle et un lieu de sociabilité pour le public.

7 L'analyse porte ici sur les 111 « bobards » proposés au vote des internautes entre 2012 et 2019 sur le site de l'évènement ${ }^{6}$, l'archivage en ligne des sélections précédentes étant incomplet. Chacun est présenté à travers une page dédiée, comprenant une synthèse plus ou moins fournie, ainsi que des supports numériques variés, liens hypertextes, vidéos, extraits audio et images, comme l'illustre l'exemple ci-dessous :

Illustration 1 : Capture d'écran de la présentation d'un " bobard ", le " bobard immigrationniste ", sur le site www.bobards-dor.fr, proposé au vote des internautes lors de l'édition 2019 des Bobards d'Or. Consulté le 15 novembre 2019.

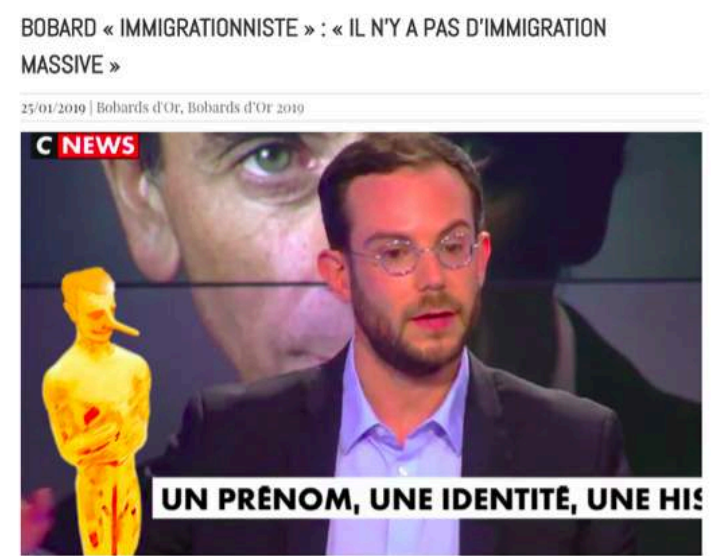

BOBARD

[Vidéo] "Immigration massive" : quand clément Viktorovitch fait voler en éclat les fake news.

Le docteur en science politique et chronique sur CNEWS Clément Viktorovitch a déconstruit brillamment les idées reçues sur limmigration massive, laissant ses contradicteurs sur la touche. Les Inrocks, 21/09/2018

RÉALITÉ

Chiffres de limmigration en hausse de 90\% par rapport à 2008. 412000 entrées légales recensées, 100000 demandeurs d'asiles, 50000 mineurs isolés. Et s'agissant des étudiants obtenant des titres de séjour, ils ne sont ni Erasmus, ni Européens pour une raison simple : avec la libre circulation, les Européens n'ont pas besoin de titres de séjour ! 
8 Le texte et les éventuels contenus présentés renvoient au média accusé de mensonge, mais aussi potentiellement à des références tierces. Nous désignons de la sorte l'ensemble des renvois à d'autres sources que le média incriminé ou à des sujets autres que celui dont la couverture est estimée mensongère. Aussi, l'analyse, qui repose ici sur un travail de codage qui s'est déroulé en deux temps. Dans le premier, le "bobard " a été l'unité d'analyse et plusieurs dimensions ont été codées, notamment la nature du média désigné comme mensonger et la mention éventuelle du nom du menteur. Un codage thématique a aussi été mis en place, permettant d'identifier ceux des «bobards » qui relèvent de la critique sociale ultra-conservatrice, caractérisée par la prégnance de schèmes identitaristes, «dessinant des identités compactes, figées et fermées" (Corcuff, 2019), valorisant des positions ultra-conservatrices et s'opposant, en conséquence, à l'Islam, à l'immigration, ou encore aux minorités ethniques et de genre. La moitié de notre corpus de 111 bobards s'insère dans cette logique. Nous n'avons pas codé l'ensemble des thématiques représentées dans la seconde moitié, celles-ci étant trop éclatées pour être agrégées dans des catégories pertinentes. Dans un second temps, les références tierces figurant dans les 111 «bobards » $(n=338)$ ont été codées, en tenant compte de la nature des sources référencées, de la variété de formes que peuvent prendre ces références et de la pluralité de sens qu'elles peuvent revêtir, en nous inspirant d'une étude menée sur les pages Facebook de Pegida (Haller et Holt, 2018). L'analyse sera par ailleurs agrémentée d'extraits des descriptions des bobards présentés sur le site Internet de l'événement. Cette analyse de contenu en ligne a été complétée par trois observations ethnographiques à la cérémonie des Bobards d'or (en 2018, 2019 et 2020) et quatre entretiens avec des responsables de sites de réinformation (TV Libertés, OJIM, Boulevard Voltaire, Breizh Info) ${ }^{7}$ conduits au printemps 2018.

9 Dans une approche inspirée de H. Becker (1985) et E. Goffman (1975), nous étudions ici les médiactivistes de réinformation comme un groupe déviant au sein de l'ensemble des acteurs produisant de l'information d'actualité. Une telle étiquette ne renvoie pas à un jugement ou une appréciation que nous porterions sur l'identité des acteurs animant les sites de réinformation ou sur la qualité des contenus qu'ils proposent : elle renvoie plutôt à un processus de catégorisation des médiactivistes de réinformation par les acteurs engagés dans une lutte de classement autour de l'énonciation de la vérité (Farkas \& Schou, 2018). En effet, les médiactivistes de réinformation se voient opposer le reproche de ne pas respecter les règles élémentaires du journalisme, que cette transgression soit avérée ou non, en faisant fi des règles d'objectivité et en diffusant essentiellement des rumeurs ou supposées fake news. Ces représentations sont essentiellement entretenues par les journalistes des médias dominants qui ont notamment popularisé l'expression "fachosphère " pour désigner les médiactivistes se réclamant de la réinformation (Albertini \& Doucet, 2016 ; Schmelck, 2017). Ainsi les associent-ils volontiers, notamment dans leurs outils de fact-checking et de certification des sites Internet, aux acteurs publiant et faisant circuler des "théories du complot » en ligne. L'appel de la réinformation à une opération de dévoilement évoque bien l'existence d'un écart, propre à la pensée dite "complotiste», entre "la réalité de surface, apparente, mais sans doute illusoire, bien qu'elle ait un statut officiel [et] une réalité profonde, cachée, menaçante, officieuse, mais bien plus réelle (Boltanski, 2012 : 15). Pour autant, postuler que ceci conduit nécessairement à la production de fake news apparaît à bien des égards contestable (Holt, 2019). Ce qui distingue cependant le groupe déviant étudié ici est la revendication d'une subjectivité militante, bousculant le principal mythe fondateur de l'illusio journalistique: le journalisme 
neutre, objectif, énonciateur de vérité. Cette objectivité journalistique a ceci de particulier qu'elle ne se limite pas à cette fonction de mythe : elle est au moins autant norme professionnelle que «rituel stratégique» (Tuchman, 1972), ensemble de pratiques et de standards "à visée normative » (Schudson, 2001). À ce titre, elle est productrice d'effets, tant sur les journalistes que sur ceux qui dévient de cette norme. C'est précisément cette démarche qui mène le groupe déviant à être perçu comme outsider au sens béckerien par une partie du champ journalistique, et notamment parmi les journalistes chargés du fact-checking.

Dans la perspective développée par H. Becker, les outsiders - dans notre cas, les médiactivistes de réinformation - peuvent ne pas reconnaître la transgression qu'on leur reproche, auquel cas ils développent une "idéologie systématique expliquant pourquoi ils sont dans le vrai et pourquoi ceux qui les désapprouvent et les punissent ont tort» (Becker, 1985: 27). C'est cette dimension que nous proposons ici de développer, en observant comment s'articulent dans le discours des médiactivistes de réinformation la revendication d'une identité militante et l'ambition d'exister en tant que réseau journalistique propre. À travers l'étude des opérations de légitimation et de délégitimation auxquelles se livre ce courant, la question centrale semble finalement être celle de la relation entretenue avec l'objet de leur critique : les médias dominants. La démarche des médiactivistes de réinformation s'appuie en effet sur des manquements éthiques et professionnels reprochés aux médias dominants, qu'ils soient constatés ou suspectés. Comment expliquer qu'ils se réclament pour autant de valeurs et de standards qui relèvent du journalisme dominant, incarnant ici la norme dont ils sont accusés de dévier? L'analyse conjointe des registres de légitimité revendiqués par les acteurs de la réinformation, et de la nature des relations qu'ils entretiennent avec le journalisme professionnel permettent de mettre en lumière les usages stratégiques de leur statut d'outsiders : tout en fondant leur existence sur la critique du journalisme professionnel (I), les acteurs de la réinformation situent clairement leur pratique dans sa grammaire et ses normes (II).

\section{Une déviance informationnelle construite contre l'Autre}

11 Toute construction identitaire se constitue sur la base d'une opposition entre un endogroupe («nous») et un exogroupe («eux»). Cette dichotomie est d'autant plus prégnante au sein de l'extrême droite qu'une conception manichéenne tend à s'imposer, distinguant l'endogroupe de la figure de l'Autre, essentialisée et diabolisée (Froio, 2017). Cette dynamique rejoint de plus celle observée par H. Becker : alors que les déviants sont « tenus pour étrangers par le reste de la société, [...] ils ont eux-mêmes tendance à considérer que le reste de la société leur est étranger » (1985 : 191). Nous proposons donc ici d'observer comment sont construits et opposés endogroupe et exogroupe, en nous appuyant sur nos analyses quantitatives et qualitatives. L'ambition parodique et humoristique de la cérémonie des Bobards d'Or permet en effet de distinguer deux groupes (Mercier, 2001). Celui des rieurs, d'abord, réunis pour réaffirmer et raffermir une identité commune. Ensuite, celui des Autres, les objets de la moquerie, envers lesquels le rire est une forme de canalisation de l'agressivité (Feuerhahn, 2001). 


\section{Usages du rire contre les « médias du système »}

12 La cérémonie des Bobards d'Or place les journalistes comme les premiers objets du rire, en tant qu'incarnations du système médiatique décrié. Plus de la moitié des «bobards» $(56,8 \%)$ mentionne ainsi nommément l'auteur du mensonge soumis au vote.

Tableau 1: Nature du média pointé du doigt par les organisateurs de la cérémonie comme responsable du "bobard"

\begin{tabular}{|l|l|l|}
\hline Nature du média & Proportion (\%) & Effectifs (n) \\
\hline TV & 30,63 & 34 \\
\hline Presse & 29,73 & 33 \\
\hline Radio & 17,12 & 19 \\
\hline Agence de presse & 12,61 & 14 \\
\hline Plusieurs médias / ensemble des médias & 8,11 & 9 \\
\hline Blogs, pure players et réseaux sociaux & 1,80 & 2 \\
\hline Ensemble & 100 & 111 \\
\hline
\end{tabular}

13 Mais au-delà des seuls journalistes, il s'agit ici de moquer le libéralisme culturel. Toute critique des médias s'insère en effet dans une perspective politique plus large (Couldry et Curran, 2003). Ici ce sont les élites culturelles, artistiques et intellectuelles qui sont visées.

«Tu es de gauche, tu es généreux, tu as un grand appartement, alors toi aussi suis les conseils de Josiane Balasko, Cécile Duflot, Esther Benbassa ou encore Jean-Luc Mélenchon, adopte un clandestin chez toi. Parce qu'en fait ils sont d'accord pour qu'ils viennent en France, mais pas chez eux. » [Extrait de la cérémonie des Bobards d'Or, animateur de la cérémonie, 10 mars 2015, Paris $\left.{ }^{8}\right]$

14 La critique de l'engagement de ces élites s'appuie notamment sur trois grandes thématiques récurrentes dans la critique sociale ultra-conservatrice (Corcuff, 2019) : immigration, islam et minorités sexuelles et de genre.

$15 \mathrm{Au}$ sein de notre corpus, $19,8 \%$ des 111 bobards traitent de la question migratoire. En riant de certaines déclarations de personnalités publiques relatives à l'immigration, les organisateurs de la cérémonie cherchent à contredire les représentations «bienpensantes" qui seraient hégémoniques dans les médias, et à réaffirmer certains éléments de leur critique. Sont ainsi estimées ridicules, et donc moquées, les déclarations présentant l'immigration comme une nécessité démographique. Le rire permet ici d'affirmer la représentation de l'immigration comme vectrice de déstabilisation et de danger, au plan économique comme au plan sécuritaire: les médias mentent et dissimulent cette menace, adoptant ainsi une posture qualifiée « d'immigrationniste ».

16 Les bobards ayant pour thématique première l'islam représentent 9,9\% des choix proposés au vote. Deux représentations sont associées à la thématique: le 
communautarisme et le terrorisme. La première s'appuie notamment sur les références à la nourriture halal et aux cafés prétendument fermés aux femmes, interprétés comme les manifestations de la volonté des Français de confession musulmane de se soustraire à la communauté nationale. La représentation de l'islam renvoie alors aux figures de l'ennemi observées par M. Jacquemain (2015) dans son étude des textes des animateurs du site Riposte Laïque. D’un côté, les musulmans de France constituent les « ennemis du bas », manifestant leur volonté de domination par l'emploi de certains signes comme le port du voile ou la consommation de nourriture halal. De l'autre, les « ennemis du haut, à savoir les intellectuels victimes de leur "bien-pensance », de leur "repentance " et de leur naïveté (...) [qui] symbolisent tout le mépris et l'arrogance des élites bienpensantes envers une grande majorité des Français qui, elle, a bien compris que l'islam est un danger " (Jacquemain, 2015 : 191). La seconde dimension à laquelle est rattaché le thème de l'islam est le terrorisme. Ainsi, tous les bobards concernant la couverture d'attentats terroristes ont pour thème sous-jacent la question de l'islam, avec notamment une dénonciation récurrente: le refus des journalistes de reconnaitre le signe de l'islamisme radical dans la couverture de ces événements.

La troisième thématique, relative à l'homosexualité et aux minorités de genre, est présente dans $7,2 \%$ des bobards. Elle fait l'objet d'une sélection spécifique en 2014, avec la remise d'un "Gay Bobard ", décrit par les organisateurs comme "un bobard militant ». Ici, c'est la posture dite "homosexualiste» des journalistes et des médias qui est dénoncée, le terme renvoyant à la fois à la survalorisation ressentie des identités homosexuelles et à la couverture estimée abusive des violences homophobes. Plus généralement, toute initiative prise en faveur d'une reconnaissance des minorités sexuelles et de genre et d'une affirmation de l'égalité entre femmes et hommes est jugée excessive car menaçant les structures élémentaires des sociétés humaines, comme le mariage ou la famille.

Aussi, le rire dirigé vers les journalistes à l'occasion de cette cérémonie est à entendre comme un rire dirigé vers les tenants du libéralisme culturel, considérés comme les alliés objectifs d'identités ethniques, culturelles et sexuelles qu'ils contribueraient à rendre visibles. L'exercice du rire dans le cadre de la cérémonie des Bobards d'Or permet donc de pointer du doigt les "ennemis du haut» et les "ennemis du bas » (Jacquemain, 2015) et d'affirmer en creux des valeurs communes à l'endogroupe. Dénoncer les «meilleurs mensonges médiatiques » ne permet pas en effet pas uniquement de mettre à l'index des boucs émissaires, mais également de constituer en victimes les groupes et initiatives qui, comme les médiactivistes de réinformation, défendent une conception essentialiste de l'identité française. Les médias, présentés comme un ensemble idéologiquement et socialement homogène, sont ainsi accusés de contribuer à un déficit de représentation des idées ultra-conservatrices.

Ceci passerait notamment par le bâillonnement des individus pouvant les porter dans les médias ou à l'université (comme E. Zemmour et B. Lugan). Pour empêcher leur prise de parole, les médias n'hésiteraient pas à véhiculer de fausses informations les concernant, à déformer leurs positions et leurs déclarations ainsi qu'à remettre en question leurs compétences. Diabolisation et délégitimation seraient donc les sanctions appliquées à ceux qui s'affranchissent du politiquement correct, de la "bien-pensance » qui caractérise pour eux les médias. L'ostracisation serait alors le prix à payer d'un "parler-vrai», ceci expliquant la carence ressentie d'opinions jugées politiquement incorrectes dans les médias dits « du système ». 
déficit de représentation médiatique concernerait en premier lieu l'activisme politique, dont deux pôles peuvent être identifiés: un pôle militant, structuré et organisé (Front National, Souveraineté, Identité et Libertés (SIEL), Génération Identitaire) mentionné dans $10 \%$ des bobards, et un pôle contestataire (La Manif pour Tous, les Veilleurs, la Marche pour la Vie, Jour de colère) représenté quant à lui dans $5,4 \%$ de notre corpus. Le principal procédé manipulatoire dénoncé est l'utilisation trompeuse de données chiffrées permettant de minorer l'ampleur de la contestation et de la remise en cause du système portée par ces pôles. La couverture des mobilisations $\mathrm{du}$ second pôle présenterait aussi les activistes sous un jour anxiogène. Ainsi, un bobard dénonce la couverture de la Manif pour Tous par l'AFP", focalisée sur les «sœurs religieuses" et les "coupes de cheveux militaires", un cadrage associant la mobilisation des "pro-familles" aux catholiques traditionalistes et aux groupuscules violents de la droite radicale. La preuve d'un traitement médiatique défavorable aux mobilisations de la droite ultra-conservatrice est aussi apportée par la comparaison avec le traitement des initiatives progressistes. La supposée montée en épingle des violences racistes et homophobes, accompagnée de la minoration, voire de la négation de celles subies par les "militants nationalistes» et les "défenseurs de la famille " participeraient pour les médiactivistes de réinformation à la propagande «bienpensante».

21 La perception d'un cadrage médiatique biaisé en leur défaveur permet aux ultraconservateurs de se présenter comme un groupe persécuté par des médias le méprisant. Aux médias dominants censés accorder une place trop importante aux thèmes de l'islamophobie, de la xénophobie et de l'homophobie sont opposées deux discriminations connues par le "vrai peuple» et ignorées, occultées ou au contraire même encouragées par les médias : le racisme anti-blanc et la christianophobie.

Les thématiques évoquées à travers les différents bobards permettent donc l'affirmation d'une identité commune, signifiée à travers les indignations et les causes défendues dans la présentation des bobards. L'ancrage droitier des participants est enrichi par une posture se voulant politiquement incorrecte et donc éloignée de la «bien-pensance » hégémonique. Le choix des bobards mis en avant par les organisateurs de la cérémonie permet de manifester un attachement aux schémas familiaux traditionnels, à une culture française et chrétienne essentialisée, des valeurs que le système médiatique ne défendrait pas et contribuerait même à mettre en danger. Face à des médias menaçant directement ces fondements, les organisateurs et les participants à la cérémonie se présentent comme un groupe dissident, engagé dans une lutte relative à des valeurs nationalistes et identitaires.

\section{Faire groupe pour affirmer une identité jugée menacée}

23 Le moment de sociabilité que constitue la cérémonie des Bobards d'or pour les membres de la réinformation et leur public semble pensé pour afficher l'image d'un groupe soudé, cohésif et dynamique. En cela, elle prolonge les logiques observées en ligne, où l'usage de liens hypertextes entre les sites de réinformation permet de donner l'image d'un courant hautement structuré, et d'unir derrière un même terme un archipel de sites aux niveaux de professionnalisation et aux types d'activité très variables. Avant même que ne commence la cérémonie, les discussions de couloir 
indiquent la présence d'une poignée d'acteurs influents, et parmi eux la figure phare de J.-Y. Le Gallou, fondateur de Polémia et organisateur de la soirée.

Jean-Yves Le Gallou est respectueusement salué par tous les spectateurs qui passent. Les caméras de deux médias de réinformation sont présentes et le suivent : BVoltaire et TV Libertés. Avant que la cérémonie ne commence, je circule dans les couloirs : l'animation et l'effervescence sont saisissantes, beaucoup se saluent les uns les autres, semblent retrouver des connaissances, boivent un verre au bar du théâtre. [Notes de terrain, cérémonie des Bobards d'Or, 9e édition, 12 mars 2018, Paris]

La fin de la cérémonie est aussi l'occasion de constater un fort niveau d'interconnaissances et de familiarité interpersonnelle :

La cérémonie s'achève sous les acclamations du public. Je note quelques remarques dans la foule qui se presse à la sortie: dans un premier temps, des remarques logistiques ("il est tard, il faut prendre la route pour rentrer à Château, c'est plus d'une heure de route"), puis sur le déroulé et le contenu de la soirée, chacun revenant sur son vote et ses justifications ("moi j'ai pas voté pour Théo ${ }^{10}$, j'ai trouvé que ça allait trop loin... Alors que les migrants, là !”) ou sur les personnalités présentes (“j'ai trouvé que Jean-Yves [probablement Le Gallou] avait l'air en forme") ». [Notes de terrain, cérémonie des Bobards d'Or, 12 mars 2018, Paris]

Sur scène, les membres du jury mettent à profit chaque opportunité permettant d'afficher la cohésion du groupe des médiactivistes de réinformation. Ainsi ils se citent mutuellement à de nombreuses reprises, s'interpellent en louant systématiquement les différents médias représentés, notamment lors du moment de l'appel aux dons. Ils incarnent leur volonté d'afficher la pluralité des médias de réinformation :

"On s'est dit qu'au-delà de Polémia, il y avait beaucoup de sites, de plateformes, d'associations, de groupes qui se lancent comme nous dans la chasse aux bobards. Chacun avec un point de vue, ou un angle particulier, ou un terrain de jeu réservé. Donc une fois élus les Bobards d'or, d'argent et de bronze, on aura une séquence réservée à trois chasseurs de bobards qui viendront nous expliquer ce qu'ils font, comment ils font, et comment on peut participer à leur combat [...]. Et comme notre camp progresse de plus en plus dans la capacité à démonter l'hydre médiatique et à mettre en évidence ses mensonges, il nous paraît important d'y associer ceux qui le font quotidiennement. » [Extrait de la cérémonie des Bobards d'Or, animateur de la cérémonie, 12 mars 2018, Paris ${ }^{11}$ ]

Au-delà des propos tenus, c'est la mise en scène même de la cérémonie et la participation du public qui attestent de la volonté de faire groupe. Ceci est manifeste entre les médiactivistes de réinformation présents sur scène, mais aussi entre ces derniers et la salle, le public étant encouragé à manifester ouvertement son amusement et ses dégoûts.

Les votes sont très vivants. Une personne dans la salle lève son carton orange avant le début des votes, il se fait moquer : "regardez celui-là, il voit un carton orange il lève la main direct! Il est du MoDem! Ah bah si, je vous ai vu, vous êtes du Modem! Mais y'a pas de mal hein, c'est bien Bayrou... Vous êtes deux !" [...] "Mesdames de la salle, si vous pouviez cesser de vous éventer, vous créez de la confusion dans le vote". [...] "Là-bas, y'a un vote triple, vous êtes un quotient familial pour frauder la CAF ou quoi ?" [...] Le Bobard Yann Moix voit ses votes accompagnés de cris, la salle commence à chauffer et s'agiter. [...] Finalement, le présentateur tranche: "puisque vous êtes chauds, on va faire à l'applaudimètre". [...] Il annonce les deux finalistes, Théo et Yann Moix, et fait crier la salle : la plupart des participants se sont levés, les cris sont de plus en plus forts et fréquents. Une partie de 
la salle scande le nom de Théo. [Notes de terrain, cérémonie des Bobards d'Or, 9e édition, 12 mars 2018, Paris]

Ainsi les Bobards d'Or présentent la particularité, parmi l'ensemble des opportunités de sociabilité de la droite radicale française, de constituer un moment de mobilisation interne - fondé sur la construction identitaire commune, et le renforcement de ces liens internes. La cérémonie est aussi un moment de mobilisation externe (Venner, 2006). En effet, elle est une occasion de gagner en visibilité : accessible en ligne, l'événement bénéficie par ailleurs d'une couverture par certains médias (France Inter ${ }^{12}$, Les Inrocks ${ }^{13}$, Libération $^{14}$ ), qui viennent prendre le pouls de la radicalité qui s'y exprime. C'est donc à travers cette mise en scène fondée sur le rire et la participation du public, ainsi que par la réaffirmation de valeurs et de référents communs, que la réinformation peut être appréhendée comme réseau cohésif, au-delà même de la structure communautaire virtuelle. Cette volonté d'une cohésion de l'endogroupe n'est pas anodine, et constitue ici un indice du caractère déviant de ce courant, "engagé dans un refus collectif de l'ordre social » (Goffman, 1975 : 167). Dans une perspective interactionniste justement, la communauté constitue pour les déviants un havre dans lequel va pouvoir s'exprimer un sentiment de supériorité, et ici de mépris, à l'égard de l'exogroupe.

Aussi, l'étude de la cérémonie des Bobards d'Or permet d'éclairer plusieurs des dimensions propres au médiactivisme de réinformation. Les thématiques des bobards proposés au vote révèlent les indignations des acteurs de la réinformation et de leur public, pour qui la dénonciation des journalistes s'insère dans une opposition au libéralisme culturel et à la mise en visibilité valorisante d'identités culturelles, ethniques et sexuelles considérées comme autant de menaces pour l'identité du «vrai peuple ». L'observation des cérémonies permet de constater le rôle du rire et des sociabilités rituelles dans l'affirmation de l'endogroupe formé par les médiactivistes de réinformation et leur public contre le champ journalistique professionnel. Pourtant, la relation que les médiactivistes de réinformation entretiennent à l'objet de leur critique, la sphère médiatique dominante, ne peut être réduite à un rejet univoque : par l'inscription de sa démarche dans l'écologie de pratiques du journalisme professionnel, la réinformation semble bien caractérisée par un double registre de légitimation, militant et journalistique, dont il s'agit d'observer l'articulation.

\section{Emprunts et subversions des pratiques journalistiques professionnelles}

Au-delà de la volonté de faire groupe contre l'Autre, la stratégie des médiactivistes de réinformation est caractérisée par l'appropriation des formes de légitimité invoquées par le journalisme professionnel, pourtant objet de ses critiques. Leur double ressort de légitimation, militant et journalistique, se fonde donc sur le développement de savoirs profanes propres à la réinformation. Ces derniers reprennent à leur compte les standards éthiques et professionnels traditionnellement attribués aux journalistes professionnels, tout en cherchant à « instituer la croyance » (Boltanski et al., 1984) dans leur mensonge généralisé ${ }^{15}$. Les emprunts au journalisme sont systématiques dans toutes les dimensions de la mise en scène de soi de ces «journalistes réinformateurs », et permettent une légitimation symbolique forte. 


\section{Se vivre journalistes à travers la réappropriation terminologique}

D'abord, dans les références et le vocabulaire employés sur les sites (pages «À propos »), on trouve une réappropriation des termes classiquement employés dans le discours journalistique, marqueurs de l'excellence de la profession (Lemieux, 1992) : " faits ", " information ", " sérieux », "qualité ", "éthique ", " investigation ", " analyse ", "vérité». Quelques exemples se trouvent dans les slogans des sites étudiés: "Notre média est créé et animé par un groupe de journalistes attachés à leur indépendance, réunis par une commune passion de l'information, de l'analyse, de l'investigation, de la réflexion et du débat » (Boulevard Voltaire) ; "Notre volonté : décrire les faits, rien que les faits, et les mettre en perspective les uns avec les autres" (Breizh Info); ou encore "Dans notre démarche journalistique, nous nous attachons à poursuivre sans relâche notre quête de Vérité, quelles qu'en soient les conséquences" (Cercle des Volontaires). De la même manière, les sites et blogs abondent en ce sens, affichant des citations se référant à la Charte de Munich de 1971, dite Déclaration des droits et devoirs du journaliste, ou à de grandes figures du journalisme du XXe siècle, comme Albert Londres. C'est, finalement, la version la plus stéréotypée ou idéalisée du journalisme traditionnel qui semble reprise ici. Dans les discours des acteurs, sur scène aux Bobards ou dans les entretiens, les autres membres de la réinformation sont des "confrères"; les sites s'organisent, en tous cas sur le papier, selon une hiérarchie journalistique classique (rédacteur en chef, responsable de rubriques, etc.). L'inspiration se prolonge jusqu'à la charte graphique des sites (voir cidessous).

Illustration 2 : Capture d'écran du générique d'introduction du programme quotidien "Le Journal » de la web-télé TV Libertés, disponible sur https://www.tvlibertes.com/emission/le-journal. Consulté le 3 juillet 2020.

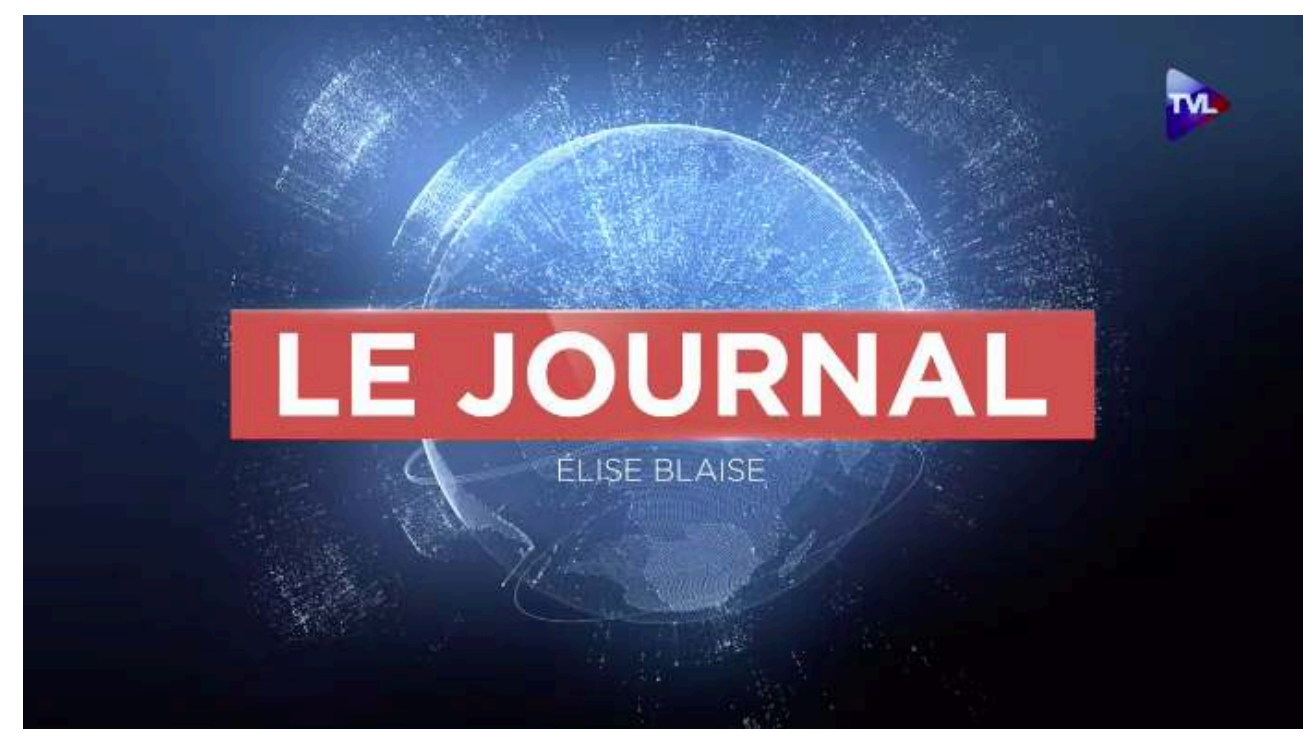

Un autre élément marque la référence au journalisme pratiqué par les médias dominants : la référence à la carte de presse. Dénoncée dans les entretiens comme une vulgaire attestation de conformisme, elle est pourtant, sur la scène des Bobards d'Or, présentée comme une marque de légitimité lorsqu'elle est mentionnée pour introduire sur scène la rédactrice en chef du seul média de réinformation " papier ", le quotidien catholique traditionaliste Présent. 
«Troisième femme du jury, elle est journaliste au quotidien Présent, eh oui, un quotidien papier, ça existe encore, c'est notre quotidien papier. Ça fait trente ans qu'elle a une carte de presse, elle a notamment différentes décorations glanées à la XVIIe Chambre Correctionnelle, notamment dès 1994, où elle est condamnée pour avoir parlé d'immigration-invasion ${ }^{16}$. Elle est aussi mère de famille, avec trois enfants [...] c'est Caroline Parmentier. » [Extrait de la cérémonie des Bobards d'Or, animateur de la cérémonie, 6 février $2017^{17}$ ] témoigne bien du fait que la réinformation se fonde sur une légitimité double, l'aspiration à se présenter comme journaliste n'allant jamais sans le rappel d'un capital militant affirmé. Ainsi, le fait d'avoir été condamnée pour diffamation publique raciale attesterait de sa «dissidence » : les institutions dénoncées - ici les «lois liberticides » étiquettent les médiactivistes de réinformation comme déviants, et permettent la validation de la dissidence dont les acteurs se revendiquent. Les stratégies des acteurs de la réinformation relevées jusqu'ici aspirent plutôt à répondre à l'étiquetage déviant les accusant de ne pas se conformer aux normes professionnelles. Anticipant des accusations de non-respect des règles élémentaires du journalisme, il s'agit pour les médiactivistes de réinformation de prouver qu'une des valeurs cardinales promue par les journalistes, l'incompatibilité entre engagement et objectivité, peut être contestée. L'exemple de la condamnation judiciaire de C. Parmentier révèle qu'une autre forme d'étiquetage déviant, cette fois apposée par l'autorité judiciaire, fait-elle aussi l'objet de resignifications. La sanction est réinterprétée ici comme le marqueur positif d'une parole exercée librement. Ce renversement est facilité par le contexte de la cérémonie, propice au rire et à l'ironie.

C'est donc bien du journalisme structuré et professionnalisé que la réinformation s'inspire. Certains vont ainsi disposer de moyens non négligeables, à l'image de TV Libertés, principale web-tv de réinformation, qui dispose de $750 \mathrm{~m}^{2}$ de locaux, composés de trois studios d'enregistrement et d'un open-space pour une quinzaine de journalistes ou stagiaires. Tous les sites d'information n'atteignent évidemment pas ce stade de développement, mais l'exemple de TV Libertés est symptomatique d'une volonté de produire un contenu revêtant les atours de ceux des médias dominants et professionnels. L'attachement à des conditions de production dignes des médias dénoncés s'insère alors dans une logique d'équivalence avec ces derniers : « Nous avons des locaux, comme vous le voyez, absolument mainstream (il rit), absolument mainstream ! » [Extrait d'entretien avec M. B., rédacteur en chef de TV Libertés, 22 mars 2018]

Cette perspective professionnelle mainstream se prolonge dans d'autres dimensions du travail de réinformation. Ainsi le rédacteur en chef de Breizh Info appuie-t-il lui aussi cette prétention à exister en tant que groupe professionnel réel : "Il manque d'ailleurs clairement un syndicat de la presse alternative, qui regrouperait les journalistes indépendants quelle que soit leur étiquette. Mais je ne désespère pas d'en fonder un ${ }^{18}$. Son média étant un site à vocation régionale, ce dernier évoque d'ailleurs son appartenance à la « $P Q R R$ » (Presse quotidienne régionale de réinformation), détournement du sigle PQR. Ce terme est pour le reste utilisé plusieurs fois au cours de la soirée des Bobards d'Or, pour inviter sur scènes des acteurs de la réinformation locale. Correspondants régionaux, $\mathrm{PQRR}$, syndicat de la presse alternative : on retrouve dans ces discours un ensemble d'éléments inspirés du journalisme traditionnel qui incarnent une forme rudimentaire de structuration d'un groupe professionnel alternatif. 


\section{Imiter et subvertir l'administration de la preuve journalistique} attendus et formels (jargon journalistique, structuration hiérarchique, détention de la carte de presse), il convient d'observer que les médiactivistes de réinformation prétendent aussi remplir les exigences propres au journalisme professionnel: l'administration de la preuve visant à énoncer la vérité, ou a minima une «obligation partielle de vérité » (Watine, 2004). Ainsi les emprunts à l'identité journalistique semblent également passer par une préoccupation importante portée à la «méthode de travail ».

Q. - Est-ce que vous diriez que justement, les membres de la rédaction ont une méthode de travail particulière par rapport à une rédaction classique?

R. - Oui, oui, oui : pour la source. En fait, oui, la source, c'est vrai que pour assurer la véracité de nos faits, ce qui compte, c'est la source. Nous avons toute une série de réseaux, d'experts, de spécialistes, de journalistes locaux, pour vraiment aller à la source, le plus possible à la source. Nous avons maintenant des correspondants locaux, avec un réseau de province, avec une grande capacité de relais, par exemple dans le cas de migrants qui s'installent dans les villages, là nous avons nous-mêmes nos propres sources, et non pas via Ouest-France ou l'AFP, ce qui permet d'avoir moins de filtre. » [Extrait d'entretien avec M. B., rédacteur en chef de TV Libertés, 22 mars 2018, Paris] serait une priorité non partagée par les médias dominants. Mais lorsqu'il développe son propos, sa définition de la source devient l'inverse exact de celle comprise dans le monde journalistique. En effet, le fait d'avoir « ses propres sources », « un réseau d'experts et de spécialistes ", revient à contourner une partie importante de ce qui est entendu et reconnu comme source par les médias traditionnels, à savoir les autres médias professionnels et les agences de presse.

La cérémonie des Bobards d'Or constitue un autre avatar de l'effort d'imitation des méthodes et outils du travail journalistique, qui emprunte les formes d'une innovation journalistique récente, le fact-checking. Que les médiactivistes de réinformation s'inspirent de cette pratique n'est pas anodin: comme l'ont démontré des travaux récents (Graves et al., 2016; Amazeen, 2015 ; Bigot, 2017), ce dispositif fondé sur la vérification transparente de faits ou de déclarations publiques permet une relégitimation de la pratique du journalisme professionnel tout en offrant aux rédactions un ressort pour se démarquer des autres médias. Sur le fond, le fact-checking dessine les contours d'une figure mythifiée: le journaliste producteur de faits parfaitement objectifs, dénué de toute subjectivité ou idéologie, fondant son travail sur des données brutes accessibles à tous et toutes, afin de produire une information profondément « orientée à la vérité » (Cornu, 1998 : 13), via des outils d'analyse dont il détient une sorte de monopole.

Instrument épistémique pur, visant à améliorer la connaissance et la diffusion de la vérité, le fact-checking joue également un rôle d'étiquetage moral. C'est justement cette deuxième fonction qui est ici investie par les acteurs. En utilisant cet outil, la cérémonie des Bobards d'Or vise à renverser la logique du fact-checking, en «factcheckant » les productions des médias dominants. Cependant, la méthode appliquée ne vise pas tant à mettre des déclarations à l'épreuve des faits pour en apprécier la véracité ou l'exactitude (Bigot, 2018), qu'à accumuler les preuves d'approximations, 
d'imprécisions ou d'omissions tirées des productions des médias professionnels, en ne cherchant pas à prouver mais à confirmer leur compromission. L'effort de fact-checking inversé réalisé lors des Bobards d'Or correspond pour les médiactivistes de réinformation à une stratégie de réappropriation de l'outil employé pour les catégoriser comme déviants. Cette démarche révèle le rapport des stigmatisés au stigmate. En reprenant les codes des dispositifs développés par ceux qui détiennent le monopole d'énonciation des normes journalistiques professionnelles, les membres de la réinformation actent l'existence d'une "différence distinctive et discréditante " (Plumauzille et Rossigneux-Méheust, 2014) avec ces derniers. Dans le même temps, ils cherchent à se légitimer à travers l'usage des normes et codes employés pour les disqualifier.

Comme on le voit ici, la prétention au fact-checking affichée par les médiactivistes de réinformation montre que les emprunts au journalisme concernent l'ensemble des ressorts d'excellence de la profession, et notamment les outils qui ont été précisément développés pour lutter contre ce que les journalistes ont appréhendé comme des déviances informationnelles et visant à conserver leur contrôle et leur autorité dans l'énonciation de l'actualité. Dans une perspective inspirée d'A. Abbott (1988), les médiactivistes de la réinformation cherchent à se saisir de l'ensemble des marqueurs symboliques de la compétence des journalistes sur leur domaine d'activité, « les atouts incopiables de la profession » (Scherer, 2011:15). On peut alors analyser la défiance croissante à l'égard des médias et l'émergence en ligne de nouveaux producteurs de contenus d'actualité comme autant d'attaques externes invitant les journalistes à affirmer leur expertise pour continuer à maîtriser leur juridiction professionnelle. Ici, la tactique adoptée par les médiactivistes de réinformation permet de mettre au jour la stratégie qui est la leur : brouiller les frontières entre médias traditionnels et médias alternatifs. Comme les journalistes professionnels, les médiactivistes de réinformation prétendent, via le fact-checking, assainir l'offre médiatique, et ainsi lutter contre les fake news. L'appui stratégique sur la confusion apparait clairement: il s'agit de contester l'autorité, en l'occurrence journalistique, en prétendant non seulement l'imiter, mais aussi jouer son rôle social à sa place.

\section{Un emploi paradoxal des références aux « médias du système »}

41 L'analyse du site Internet des Bobards d'Or révèle que près de deux tiers des «bobards " proposés au vote des internautes $(64,9 \%)$ comportent une référence à des informations ou contenus externes visant à contester le "mensonge $»^{19}$. À l'inverse, un tiers des bobards ne contient pas de références permettant de faire la preuve du mensonge, et fondent leur démonstration en s'appuyant sur un supposé "bon sens». Certaines assertions sont ainsi présentées comme frauduleuses ou illogiques, sans même que soit ressentie la nécessité d'en faire la preuve. Il peut s'agir de démonstrations faisant appel à la logique, comme ici lorsqu'il s'agit de contester l'analyse des résultats du Front national aux élections cantonales de 2011 :

"Rappelons donc à ces étranges "experts" comment est calculé un pourcentage électoral : au dénominateur, le nombre des votants; au numérateur, le nombre des suffrages exprimés en faveur d'un parti. Quand un parti augmente le nombre de ses candidats, il augmente le nombre des suffrages recueillis et donc son pourcentage global. C'est du niveau de la classe de CM1. » [Description du bobard «Calvi, Dély, Cayrol et Barbier (France 5), les champions d'arithmétique électorale sur France 5, le mensonge "C'est dans l'air !" ", site Internet, 2012 ${ }^{20}$ ] 
Dans le même temps, ce «bon sens» est fortement associé aux conceptions identitaristes du monde social exposées précédemment. La publication d'un entretien avec deux économistes présentant l'immigration comme un moyen de dynamiser l'économie française est ainsi l'occasion de les réaffirmer, en les présentant comme des évidences :

«En se focalisant sur l'aspect numérique et démographique nos "spécialistes" oublient qu'un pays n'est pas qu'une somme d'individus et que l'accroissement de l'immigration poserait un problème majeur d'intégration, que l'on ressent déjà cruellement aujourd'hui. Bel exemple de naïveté et de bêtise... » [Description du bobard, «La Tribune: la France a besoin de 10 millions d'immigrés d'ici 2040 », site Internet, 2012 ${ }^{21}$.

Comme indiqué plus haut, deux tiers des bobards convoquent une ou plusieurs preuves permettant de contester la véracité ou l'authenticité de l'assertion médiatique considérée comme un bobard. Près d'un tiers des «preuves » mobilisées sont issues de médias de réinformation ou d'organisations affiliées $(30,26 \%)$. La source la plus citée dans ce groupe est le site de l'observatoire du Journalisme (OJIM). L'activité éditoriale de ce site consiste principalement dans la production d'articles en réaction à des déclarations de personnalités publiques et de fiches ayant pour objet de renseigner l'identité, les engagements et prises de position des acteurs des médias dominants. De ce fait, il n'est pas surprenant d'observer qu'il est le site le plus employé pour remettre en question et contester les discours des médias dominants.

Tableau 2 : Distribution de la nature des sources tierces invoquées pour faire la preuve du mensonge

\begin{tabular}{|l|l|l|}
\hline Nature de la source & Proportion (\%) & Effectifs (n) \\
\hline Médias mainstream (dont AFP) & 34,21 & 52 \\
\hline Médias ou acteurs de réinformation et affiliés & 30,26 & 46 \\
\hline Sources institutionnelles (INED, INSEE, rapports ministériels) & 8,55 & 13 \\
\hline Acteurs de la critique des médias (Arrêt sur images) & 0,66 & 1 \\
\hline Autres & 26,32 & 40 \\
\hline Ensemble & 100 & 152 \\
\hline
\end{tabular}

Cependant, la source principale mobilisée pour contrer les «mensonges des médias dominants " consiste en des productions de ces mêmes médias dominants $(34,21 \%)$. Dans cette configuration, l'entreprise de réinformation est caractérisée par une forte dépendance à l'égard de ces médias, pourtant rejetés. Ce point a été précédemment souligné dans plusieurs contributions: une étude employant des méthodes computationnelles révèle en effet que les sites d'extrême droite tendent à renvoyer vers les sites d'information mainstream (Venturini et al., 2018). Des études monographiques permettent de qualifier les logiques dans lesquelles s'insèrent ces renvois: la production éditoriale de certains sites consiste essentiellement en une reformulation des articles des médias mainstream (Jammet et Guidi, 2017; Lukasik, 
2018). Une étude a également été conduite sur les pages Facebook de Pegida, en Autriche et en Allemagne, un mouvement communiquant essentiellement via les réseaux socio-numériques avec ses sympathisants (Haller et Holt, 2018). Malgré une dénonciation virulente des "médias menteurs", ces pages renvoient à ces derniers quand leurs productions peuvent être interprétées comme la confirmation des vues et opinions identitaires défendues par Pegida. Aussi les liens dirigeant vers des médias dominants depuis des sites d'extrême droite pourtant critiques à l'égard de ces derniers n'ont pas forcément une valeur contestataire, ils peuvent être des liens affirmatifs (ibid.). Ces renvois concernent notamment la presse quotidienne régionale, qui présente la qualité, aux yeux des médiactivistes de réinformation, de donner une place plus importante que les titres nationaux aux faits-divers.

Cette dépendance apparaît comme paradoxale eu égard à la mobilisation à titre de preuves de certaines sources. Ainsi l'Agence France Presse (AFP) est présentée comme «l'origine de tous les mensonges", et cumule plusieurs défauts aux yeux des médiactivistes de réinformation : elle exercerait un contrôle homogénéisateur sur les actualités, symboliserait ainsi la pensée unique médiatique, en étant financée par des fonds publics, et ne serait donc pas à la hauteur de son rôle dans la société française. "L'AFP en France fait la pluie et le beau temps, l'AFP tousse et la presse tousse, l'AFP ment et la presse ment » [Extrait de la cérémonie des Bobards d'Or, animateur de la cérémonie, 12 mars 2018]

À ce titre, $12.61 \%$ des bobards proposés au vote désignent l'AFP comme coupable, et l'agence est fréquemment représentée lors de la cérémonie par un prix qui lui est dédié (en 2015, 2016, 2017 et 2018). Pourtant, à deux reprises dans les bobards présentés sur le site, les informations produites par l'agence de presse sont mobilisées à titre de preuve, pour rétablir une vérité estimée écornée par d'autres médias professionnels. Les références aux médias dominants concernent aussi les journaux Le Monde et Libération, quand bien même les médiactivistes de réinformation les suspectent et les accusent de pratiquer un fact-checking motivé par des considérations idéologiques plus que par des standards éthiques et professionnels. Comme dans le cas susmentionné de l'AFP, il peut apparaître surprenant d'observer que des contenus produits par les Décodeurs du Monde et par Checknews de Libération sont mobilisés à titre de preuve. Ainsi, à l'occasion de la cérémonie des Bobards d'Or de 2019, Polémia souhaite dénoncer une vidéo mise en ligne par France TV Education, qui falsifierait la réalité en présentant la dépénalisation de l'homosexualité comme le résultat de l'action du gouvernement Mauroy. Les organisateurs de la cérémonie redirigent alors, par un lien hypertexte, vers un article de Checknews intitulé « L'homosexualité a-t-elle été dépénalisée en 1791 ou en 1982 ? ». La synthèse des conclusions de l'article proposée sur le site des Bobards n'en reste pas moins sélective et partielle: elle évacue notamment la permanence des discriminations contre les homosexuels après la dépénalisation du crime de sodomie en 1791, pourtant mentionnée dans l'article de fact-checking.

Les médiactivistes de réinformation empruntent donc à deux registres dans leur discours de légitimation de leur activité : militant, en revendiquant une certaine radicalité et en s'ancrant dans un clivage Eux/Nous ; et professionnel, en reprenant à leur compte les marqueurs de l'activité journalistique telle qu'elle est pratiquée au sein des rédactions des médias dominants. L'emprunt au journalisme professionnel se retrouve pleinement à l'occasion de la cérémonie des Bobards d'Or, pour laquelle les 
médiactivistes de réinformation proposent de soumettre les actualités des médias dominants à leur propre fact-checking.

Finalement, l'analyse de cette relation ambivalente au monde journalistique soulève la question de ce qui, dans la nature même du champ, permet ces emprunts. La relative facilité avec laquelle ces acteurs se déclarent journalistes nous renvoie en effet aux problématiques liées aux contours de la profession. D. Ruellan, reprenant Bucher et Strauss (1961) présente le groupe des journalistes comme « fait d'amalgames lâches de segments poursuivant des objectifs différents de manières différentes, et se tenant plus ou moins fragilement sous une dénomination commune à une période spécifique de l'histoire ». Ce «flou » ne doit pas être considéré comme un dysfonctionnement : il est un marqueur constitutif de ce groupe professionnel (Ruellan, 1993). Avec la loi de 1935 sur le statut du journaliste professionnel, c'est aujourd'hui une définition juridique tautologique qui prévaut: est journaliste celui qui tire la majorité de ses revenus du journalisme ${ }^{22}$. Dès lors, pourquoi une même lapalissade serait-elle moins recevable sur le plan symbolique ? Pourquoi le statut de journaliste ne serait-il pas reconnu à celui qui associe ses activités éditoriales au journalisme? C'est la question que soulèvent les médiactivistes de réinformation, en empruntant non seulement un ensemble de références, valeurs et lexiques relevant de la symbolique du journalisme traditionnel, mais aussi, beaucoup plus matériellement, en imitant ses logiques de structuration professionnelle.

\section{Conclusion}

En adoptant une approche empruntant à la sociologie de la déviance, nous avons ici proposé d'analyser les modalités par lesquelles les médiactivistes de la réinformation placent leurs pratiques dans la lignée de la grammaire propre au journalisme professionnel. Mis à l'index par les acteurs traditionnels de la production d'information d'actualité, les médiactivistes de réinformation et leur public tentent d'affirmer un système alternatif de normes, et construisent une légitimité fondée sur une hybridation originale entre journalisme et militantisme. Leurs espaces de sociabilité hors ligne, à l'image de la cérémonie des Bobards d'Or et de ses rituels, sont l'occasion d'exprimer leur rejet du libéralisme culturel, ainsi que leur volonté de faire groupe autour de valeurs partagées. La posture militante assumée, fondée sur une conception identitariste du monde social, s'appuie aussi sur une valorisation de la radicalité des vues et opinions exprimées. Pour autant, l'opposition au «système» et à sa "bienpensance " n'empêche pas les médiactivistes de réinformation de se s'inspirer des normes propres au système médiatique traditionnel et professionnel pour fonder la légitimité de leur entreprise. Ceci se traduit notamment par la reprise à leur compte du vocabulaire et des référents de la profession, mais aussi et surtout par la réappropriation d'une pratique contemporaine, marquant son excellence et de sa prétention à énoncer la vérité : le fact-checking.

50 Aussi, la complexité de la relation qui lie les médiactivistes de réinformation aux journalistes mainstream renvoie au rapport ambivalent qu'ils entretiennent à leur étiquetage déviant, tour à tour stratégiquement revendiqué ou nié. La volonté de se distinguer de l'objet de leur critique tout en affirmant une égale prétention à énoncer la vérité les amène à emprunter aux codes du journalisme tout en en rejetant certaines dimensions, à s'en inspirer tout en le dénonçant. C'est par l'analyse ethnographique de 
la cérémonie des Bobards d'Or, de sa mise en scène comme des discours qu'y tiennent les acteurs, que ces stratégies apparaissent clairement. Au-delà de la volonté de faire groupe autour de valeurs partagées, notamment avec le public, tout est fait pour que les acteurs apparaissant sur scène soient perçus comme des journalistes professionnels et légitimes, qui aspirent à pratiquer une forme idéalisée de journalisme. Dans cette perspective, ce sont finalement les journalistes mainstream qui dévieraient des exigences de leur groupe professionnel, renonçant à la quête de vérité du fait de leur «bien-pensance ». Ainsi, la volonté de faire état des mensonges des médias doit être vue ici comme la manifestation de la volonté des médiactivistes de réinformation d'instituer la croyance en un paysage médiatique compromis par le mensonge et la dissimulation. La logique à l'œuvre ici est conforme à celle observée par L. Boltanski dans Énigmes et complots (2012): l'existence de précédents à l'occasion desquels le mensonge de certaines autorités est avéré permet l'instillation du doute et encourage l'adhésion à des schèmes explicatifs alternatifs à ceux proposés par ces mêmes autorités. C'est donc quand l'acceptabilité des discours officiels, ici celle des médias mainstream, est compromise que des contre-discours prétendant à la vérité peuvent émerger, se renforcer, jusqu'à revendiquer une inscription pleine et entière dans le champ journalistique.

\section{BIBLIOGRAPHIE}

Аввотт Andrew Delano (1988). The system of professions: an essay on the division of expert labor, Chicago, University of Chicago Press.

ALBERTINI Dominique \& DOUCET David (2016). La fachosphère : comment l'extrême droite a remporté la bataille d'Internet, Paris, Flammarion.

ALDUY Cécile \& WAHNICH Stéphane (2015). Marine Le Pen prise aux mots : décryptage du nouveau discours frontiste, Paris, Éditions du Seuil.

AMAZEEn Michelle A. (2015). « Revisiting the epistemology of fact-checking », Critical Review, 27 (1), pp. 1-22.

BECKER Howard S. (1985). Outsiders, Paris, Éditions Métailié.

BIGOT Laurent (2017). « Le fact-checking ou la réinvention d'une pratique de vérification », Communication \& langages, 192 (2), pp. 131-156.

BIGOT Laurent (2018). « Rétablir la vérité via le fact-checking : l'ambivalence des médias face aux fausses informations ", Le Temps des medias, 30 (1), pp. 62-76.

BOLTANSKI Luc (2012). Énigmes et complots : une enquête à propos d'enquêtes, Paris, Gallimard.

BOLTANSKI Luc, DARRÉ Yann \& SCHILTZ Marie-Ange (1984). « La dénonciation », Actes de la Recherche en Sciences Sociales, 51 (1), pp. 3-40.

BOLTANSKI Luc \& ESQUERRE Arnaud (2014). Vers l'extrême : extension des domaines de la droite, Paris, Éd. Dehors. 
BUCHER Rue \& STRAUSS Anselm (1961). «Professions in process », American Journal of Sociology, 66 (4), pp. 325-334.

CAMUS Jean-Yves (1996). L'extrême droite aujourd'hui, Toulouse, Milan.

CAMUS Jean-Yves \& MONZAT René (1992). Les droites nationales et radicales en France : répertoire critique, Lyon, Presses universitaires de Lyon.

CARDON Dominique \& GRANJon Fabien (2010). Médiactivistes, Paris, Presses de Sciences Po.

CORCUFF Philippe (2019). « De la rebellitude ultra-conservatrice et ce qu'elle fait aux sciences sociales critiques », Sociologies [en ligne], consulté le 09.04.21. URL : http://

journals.openedition.org/sociologies/10462

CORNU Daniel (1998). « Journalisme et la vérité », Autres Temps. Les cahiers du christianisme social, 58 (1), pp. 13-27.

COULDRY Nick \& CURRAN James (2003). « The paradox of media power », in Couldry Nick (ed.), Contesting media power : alternative media in a networked world. Lanham, Rowman \& Littlefield, pp. 3-15.

FARKAS Johan \& SCHOU Jannick (2018). « Fake news as a floating signifier: hegemony, antagonism and the politics of falsehood ", Javnost - The Public, 25 (3), pp. 298-314.

FEUERHAHN Nelly (2001). « La dérision, une violence politiquement correcte », Hermès, 29, pp. 187-197.

FIGENSCHOU Tine Ustad \& IHLEBÆK Karoline Andrea (2018). « Challenging journalistic authority: media criticism in far-right alternative media », Journalism Studies, 20 (9), 1221-1237.

FRoIO Caterina (2017). « Nous et les autres. L'altérité sur les sites web des extrêmes droites en France », Réseaux, 202-203 (2-3), pp. 39-78.

GOFFMAN Erving (2015). Stigmate : les usages sociaux des handicaps (Alain Kihm, Trans), Paris, Minuit. GRAVES Lucas, NYHAN Brendan \& REIFLER Jason (2016). « Understanding innovations in journalistic practice: a field experiment examining motivations for fact-checking: Understanding innovations in journalistic practice ", Journal of Communication, 66 (1), pp. 102-138.

HALLER André \& HOLT Kristoffer (2019). « Paradoxical populism: how PEGIDA relates to mainstream and alternative media », Information, Communication \& Society, 22 (12), pp. 1665-1680.

HOLT Kristoffer (2019). Right-wing alternative media. London, Routledge.

JACQUEMAIN Marc (2015). « La laïcité réactionnaire : une tentative de déconstruction », in Durand Pascal \& Sindaco Sarah (eds.), Le discours « néo-réactionnaire » : transgressions conservatrices, Paris, CNRS éditions, pp. 185-198.

JAMMET Thomas \& GUIDI Diletta (2017). « Observer Les Observateurs. Du pluralisme médiatique au populisme anti-islam, analyse d'un site de « réinformation » suisse et de ses connexions ", Réseaux, 202-203 (2-3), pp. 241-271.

KOROLITSKI Ulysse (2015). Punir le racisme. Liberté d'expression, démocratie et discours racistes, Paris, CNRS Editions.

LEMIEUX Cyril (1992). « La Révolution française et l'excellence journalistique au sens civique. Note de recherche », Politix, 5 (19), pp. 31-36. 
LUKASIK Stéphanie (2018). «A la frontière des « fake news », entre réinformation et

désinformation, le cas du blog Fdesouche », in Joux Alexandre \& Pélissier Maud (dir.), L'information d'actualité au prisme des « fake news », Paris, Harmattan, pp. 135-158.

MERCIER Arnaud (2001). « Pouvoirs de la dérision, dérision des pouvoirs », Hermès, 29, pp. 9-18.

PLUMAUZILLE Clyde \& ROSSIGNEUX-MÉHEUST Mathilde (2014). « Le stigmate ou « La différence comme catégorie utile d'analyse historique » ", Hypothèses, 17 (1), pp. 215-228.

REBILLARD Franck (2007). Le Web 2.0 en perspective : une analyse socio-économique de l'Internet, Paris, L'Harmattan.

REBILLARD Franck \& SMYRNAIOS Nikos (2010). « Les infomédiaires, au cœur de la filière de l'information en ligne. Les cas de Google, Wikio et Paperblog ", Réseaux, 160-161 (2-3), pp. 163-194.

RUELLAN Denis (1993). Le professionalisme du flou : identité et savoir-faire des journalistes français. Grenoble, Presse Univ. de Grenoble.

SCHERER Éric (2011). A-t-on encore besoin des journalistes? Manifeste pour un journalisme augmenté, Paris, Presses Universitaires de France.

schmelck Clara (2017). «Plongée en fachosphère », Médium, 52-53 (3-4), pp. 199-212.

SCHUDSON Michael (2001). « The objectivity norm in American journalism », Journalism, 2 (2), pp. 149-170.

TUCHMAN Gaye (1972). « Objectivity as strategic ritual: an examination of newsmen's notions of objectivity », American Journal of Sociology, 77 (4), pp. 660-679.

UNRUH David R. (1980). « The nature of social worlds », Pacific Sociological Review, 23 (3), pp.

271-296.

VENNER Fiammetta (2006). Extrême France : les mouvements frontistes, nationaux-radicaux, royalistes, catholiques traditionalistes et provie, Paris, Bernard Grasset.

VENTURINI Tommaso, JACOMY Mathieu, BOUNEGRU Liliana \& GRAY Jonathan (2018). « Visual network exploration for data journalists ", in Eldridge Scott \& Franklin Bob (dir.), The Routledge handbook of developments in digital journalism studies. Londres, Routledge, pp. 265-283.

WATINE Thierry (2004). « Entre réalité, vérité et objectivité : la "perspective” journalistique », Les Cahiers du journalisme, 13, pp. 24-39.

\section{NOTES}

1. Nous employons ici le néologisme forgé par D. Cardon et F. Granjon pour désigner «les mobilisations sociales qui orientent leur action collective vers la critique des médias dominants et/ou la mise en œuvre de dispositifs alternatifs de production d'information » (2010: 8). Si les acteurs ici présentés ne relèvent pas des courants progressistes étudiés par les deux auteurs, ils partagent avec ces derniers un répertoire d'action : critique de l'ordre dominant de l'information et mise en place de circuits d'information. Aussi nous les qualifierons dans cet article de médiactivistes de réinformation.

2. Cet énarque, membre fondateur du Club de l'Horloge, cercle de réflexion ayant pour objet d'influer idéologiquement la haute administration, rejoint le Front National en 
1985. Artisan de la scission mégrétiste en 1998, il compte parmi les cadres du Mouvement national républicain dont il s'éloigne au début des années 2000 pour se concentrer sur son think-tank identitaire Polémia.

3. Le terme est employé de façon récurrente dans les productions de Polémia. L'évocation de cette entité laissée floue permet dans les discours d'extrême droite d'évoquer une intentionnalité cachée (Alduy \& Wahnich, 2015) mais aussi de conjuguer imaginaires contestataires et complotistes (Boltanski \& Esquerre, 2014).

4. L'expression est employée à la fois par les médiactivistes progressistes et les médiactivistes de réinformation, bien que ces derniers utilisent aussi des expressions qui leur sont plus propres idéologiquement: médias "centraux ", "subventionnés ", «de l'oligarchie ». Par commodité, nous employons l'expression dans l'article pour distinguer ces médias des médias de réinformation.

5. Présentation de la cérémonie sur son site Internet. Accès : https://bobards-dor.fr/

6. Disponibles sur le site https://bobards-dor.fr/.

7. Entretien avec M. B., rédacteur en chef de TV Libertés, 22 mars 2018 ; Entretien avec C. C., président de l'Observatoire du Journalisme, 28 mars 2018 ; Entretien avec G. C., rédactrice en chef de Boulevard Voltaire, 18 mai 2018 ; Échanges de mails avec Y. V., rédacteur en chef de Breizh Info, 5 au 29 avril 2018.

8. « 6 e cérémonie des Bobards d'Or de la Fondation Polémia ", Dailymotion, vidéo publiée par Agence2Presse. 17300 vues au 28/08/2020. Accès: https:// www.dailymotion.com/video/x2hrrj8. A partir de 42'00.

9. "Le bobard "calculette" ", cérémonie des Bobards d'Or 2013. Accès: http:// www.bobards-dor.fr/2013/1572/archives.

10. «L'Affaire Théo » renvoie à des faits produits en février 2017 : l'arrestation par les forces de l'ordre d'un jeune homme à l'occasion de laquelle ce dernier avait été blessé par un coup de bâton télescopique à l'anus, entraînant une incapacité temporaire de travail de 60 jours.

11. «Bobards d'Or 2018 - La cérémonie intégrale », YouTube, vidéo publiée par Les Bobards d'Or. 12468 vues au 1er septembre 2020. Accès : https://www.youtube.com/ watch?v=hvtC-i3vR4c. À partir de 5'00.

12. Meurice G., «La réinformation », Le moment Meurice, France Inter, 7 février 2017. Accès : https://www.franceinter.fr/emissions/le-moment-meurice/le-momentmeurice-07-fevrier-2017, Consulté le 25 novembre 2019.

13. Thoreau C., «Ce qui s'est dit aux Bobards d'Or 2018, le défouloir anti-média de l'extrême droite", Les Inrockuptibles, 13 mars 2018. Accès : https:// www.lesinrocks.com/2018/03/13/actualite/medias-actualite/ce-qui-sest-dit-aux-

bobards-dor-2018-le-defouloir-anti-media-de-lextreme-droite/. Consulté le 19 nov. 2019.

14. Albertini D., "La fachosphère se paie la tournée des bobards ", Libération, 9 mars 2015. Accès : https://www.liberation.fr/france/2015/03/09/la-fachosphere-se-paie-latournee-des-bobards_1217512. Consulté le 15 novembre 2019.

15. Si la cérémonie se concentre sur une sélection de prétendus mensonges, le discours développé par la Fondation Polémia et son principal représentant, J.-Y. Le Gallou, ne laisse pas de doute sur la généralisation de la critique. Ainsi, "pour défendre le 
politiquement correct sous toutes ses formes, les journalistes désinforment leur public » (La Tyrannie médiatique, J.-Y Le Gallou, p.159).

16. Le 9 février 1994, la LICRA fait citer Caroline P. devant le Tribunal correctionnel de Paris pour diffamation et injure raciales, provocation à la discrimination, à la haine et à la violence raciale, suite à un article dans Présent, dans lequel elle décrivait les immigrés comme «trop nombreux et trop inassimilables ». Elle fut déclarée coupable de diffamation publique raciale. Pour une étude plus exhaustive de ce cas juridique, on se référera à Korolitski (2015: 157-158).

17. «Les Bobards d'Or 2017 », YouTube, vidéo publiée par Les Bobards d'Or. Accès : https://www.youtube.com/watch?v=F8_gu9EOoYE\&ab_channel=LesBobardsd\%27or. À partir de 8'00. On peut également noter dans cet extrait la revendication du statut de «mère de famille ». Comme le discours tenu sur «l'idéologie féministe » le confirme plus tard dans la soirée, il est important pour ces acteurs de revendiquer ce que justement, le « système bien-pensant » exclut, selon eux : le fait de définir la femme par son statut de mère.

18. Echanges par mail avec Y.V., rédacteur en chef de Breizh Info, 5 avril 2018.

19. $45,1 \%$ des références tierces entrent dans cette logique. Les autres fonctions sont l'exposé de la circulation du «bobard» $(28,8 \%)$, l'illustration de la compromission idéologique des journalistes $(15,4 \%)$ et la contextualisation du «bobard» (10,7\%). Une seule référence n'a pas pu faire l'objet d'un codage, elle correspond à un lien YouTube cassé.

20. Accès : http://www.bobards-dor.fr/2012/portfolio/calvi-dely-cayrol-et-barbierfrance-5/

21. Accès : http://www.bobards-dor.fr/2012/portfolio/la-tribune/

22. Aussi appelée Loi Brachard, du nom du député et journaliste porteur du projet, elle établit la base du statut de la profession, aujourd'hui contenue dans l'article L.7111-3 du Code du Travail : «Est journaliste professionnel toute personne qui a pour activité principale, régulière et rétribuée, l'exercice de sa profession dans une ou plusieurs entreprises de presse, publications quotidiennes et périodiques ou agences de presse, et qui en tire le principal de ses ressources ».

\section{RÉSUMÉS}

Le courant de la "réinformation» désigne un groupe d'acteurs militants alliant un ancrage idéologique identitaire et un répertoire d'action fondé sur une pratique informationnelle. Ainsi leur existence se fonde-t-elle sur une prétention à énoncer " la vraie vérité ", tout en dénonçant ceux qui, selon eux, trahiraient les exigences professionnelles et éthiques du journalisme : les médias «mainstream». L'étude ici proposée se concentre sur un moment de sociabilité important pour le mouvement: les Bobards d'Or, une cérémonie annuelle parodique récompensant «[les journalistes] qui n’hésitent pas à mentir délibérément pour servir le politiquement correct », organisée à Paris par la Fondation Polémia. En croisant l'observation ethnographique de plusieurs éditions de la cérémonie, et une analyse statistique des 111 
«mensonges » proposés au vote des internautes entre 2012 et 2019 , nous analysons les modalités par lesquelles ces acteurs construisent une légitimité hybride, affichant des positions militantes tout en se réclamant de la grammaire du journalisme professionnel. Par un usage stratégique de leur statut d'outsiders, dans leurs discours et dans leurs pratiques, les réinformateurs cherchent à inscrire leurs pratiques comme conformes aux valeurs et standards du journalisme dit « mainstream ». Ce faisant, ils prétendent endosser les normes dont ils sont accusés de dévier.

The "reinformation" movement designates a group of far-right activists that developed an informational practice while maintaining a strong ideological identity. They justify their existence through a claim on "the real truth", as they denounce the "mainstream" media they depict as betraying the professional and ethical requirements of journalism. This study focuses on an important moment of sociability for the movement: the "Golden Lies" (Bobards d'Or) ceremony, that rewards "[journalists] who do not hesitate to deliberately lie to serve political correctness". By combining ethnographic observations of several editions of the ceremony, and a statistical analysis of the 111 "lies" selected by the organizers between 2012 and 2019, we investigate the modalities by which these activists construct a hybrid legitimacy. Through a strategic use of their outsider status, re-informers seek to inscribe their practices as consistent with the grammar of professional journalism. In doing so, they claim to endorse the norms from which they are accused of deviating.

\section{INDEX}

Keywords : journalism, far-right activism, identity politics, re-information, disinformation studies

Mots-clés : réinformation, médiactivisme, extrême-droite identitaire, journalisme

\section{AUTEURS}

\section{GAËL STEPHAN}

Université Paris 2 Panthéon-Assas, CARISM

\section{YSÉ VAUCHEZ}

Université Paris I Panthéon-Sorbonne, CESSP/CRPS 